\title{
Sputum completion and conversion rates after intensive phase of tuberculosis treatment: an assessment of the Rwandan control program
}

Felix R Kayigamba ${ }^{*}$, Mirjam I Bakker², Veronicah Mugisha ${ }^{3}$, Michel Gasana ${ }^{4}$ and Maarten F Schim van der Loeff 5,6

\begin{abstract}
Background: In Rwanda tuberculosis (TB) is one of the major health problems. To contribute to an improved performance of the Rwandan National TB Control Program, we conducted a study with the following objectives: (1) to assess the completion rate of sputum smear examinations at the end of the intensive phase of TB treatment; (2) to assess the sputum conversion rate (SCR); (3) to assess associations between smear completion rate or SCR with key health facility characteristics.

Methods: TB registers in 89 health facilities in five provinces were reviewed. Data of new and retreatment smearpositive pulmonary TB (PTB+) cases registered between January and June 2006 were included in the study. Data on key characteristics of the selected health facilities were also collected.

Results: Among 1509 new PTB + cases, 32 (2.1\%) had died by 2 months, and 178 (11.8\%) had been transferred-out. Among the remaining 1299 patients, a smear examination at month 2 was done in 1039 (smear completion rate 80.0\%). Among these 1039, 852 (82.0\%) had become smear-negative. The smear completion rate and SCR varied considerably between health facilities. A high number of new PTB cases at a health facility was the only significant predictor of a low completion rate, while the only independent factor associated with low sputum conversion rates was rural (vs. urban) location of the health facility.

Conclusions: In Rwanda, too few patients get a smear examination after 2 months of TB treatment; the SCR among those with smear results was adequate at $82 \%$. A high number of new TB patients at a health facility was a significant predictor of a low completion rate. The national TB control program should design strategies to improve completion rates.
\end{abstract}

Keywords: Sputum smear examination, Mycobacterium tuberculosis, Pulmonary tuberculosis, Africa

\section{Background}

A third of the world's population is infected by Mycobacterium tuberculosis and it is estimated by the World Health Organization (WHO) that 1.45 million people die each year from tuberculosis (TB) [1]. Ninety-five percent of TB cases and 98\% of TB deaths occur in developing counties [2]. In Rwanda TB is a major health problem: in 2010 the TB notification rate was 72 per 100,000 population [3].

The sputum conversion rate (SCR) is the percentage of smear-positive pulmonary $\mathrm{TB}(\mathrm{PTB}+)$ cases registered

\footnotetext{
* Correspondence: fkaigamba@gmail.com

'INTERACT, CPCD, PO Box 2181, Kigali, Rwanda

Full list of author information is available at the end of the article
}

in a specified period that converted to smear negative status after the standard two months of the intensive phase of treatment. WHO recommends its use as a useful indicator for TB control programs in monitoring the TB program performance, and as a trigger for rigorous assessment in patients with still positive smears [4]. Even in well functioning national TB programs $25 \%$ of initially PTB + patients may still be smear-positive at the end of the intensive phase of treatment, despite good adherence and supervised medication [5].

Once the sputum smear has become negative, patients are considered to be no longer infectious [6]. Those failing to achieve sputum conversion after 2 months are more likely to have poor treatment outcomes [7-10]. If

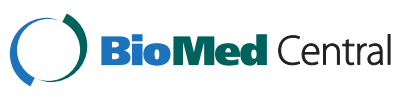


the sputum smear is still positive after 2 months of treatment, the intensive phase is extended for one more month $[5,11]$.

Studies to evaluate the SCRs and the predictive factors for failure to convert have been conducted in several countries in sub-Saharan Africa and elsewhere, but not in Rwanda [7-10,12-23].

The objectives of this study were to assess the proportion of patients in Rwandan health facilities for whom a smear examination after the intensive phase of treatment was done (completion rate), to assess the sputum smear conversion rate among new cases and retreatment PTB + cases, and to assess whether key health facility characteristics are associated with smear completion rate or with SCR in the Rwandan program.

\section{Methods}

\section{Procedures}

We conducted a review of $\mathrm{TB}$ treatment registers in health facilities. The diagnosis of PTB in Rwanda is mainly by sputum microscopy. The Kinyoun staining technique was used during the study period (in 2009 Ziehl-Neelsen staining was adopted). All patients attending out-patient departments with cough for three weeks or longer, were considered TB suspects and requested to submit three sputum samples for microscopic examination (usually on two consecutive days). At least two positive smears were required to confirm a suspected TB case as PTB + [24] (in 2009 guidelines were changed to two weeks of cough and at least one positive smear) [11].

\section{Treatment Regimen}

In Rwanda, TB treatment is directly supervised. The treatment regimen for new PTB + cases in Rwanda consists of a two month intensive phase with a daily dose of fixeddose combination tablets containing Rifampicin (R), Isoniazid $(\mathrm{H})$, Pyrazinamide $(\mathrm{Z})$ and Ethambutol $(\mathrm{E})$, followed by a four months continuation phase of daily $\mathrm{RH}$. To assess sputum conversion, sputum microscopy is done after the second (C2), fourth and sixth month of treatment. $\mathrm{PTB}+$ cases are expected to convert to negative sputum status after two months of intensive TB treatment.

TB retreatment cases include cases that relapsed after, defaulted during or failed on first line treatment. These are treated with an eight-month regimen consisting of daily streptomycin (S) and RHZE for two months, daily RHZE for one month, and daily RHE for five months. Sputum microscopy to assess conversion is done after 3 months of intensive treatment [11].

\section{Study setting}

To achieve representativeness for Rwanda, this study was performed in three administrative districts selected from each of the five provinces. Kigali city has three districts while the other four provinces have more. All three Kigali city districts were included in the study while the three districts with the highest numbers of patients registered during the period we studied were selected from each of the other four provinces. All TB diagnostic health facilities $(n=89)$ in the selected 15 districts were included in the study.

\section{Data collection and management}

At each selected health facility aggregated data of TB patients registered during the study period (January-June 2006) were abstracted. Data included the numbers of patients whose sputum was not evaluated, who converted to negative sputum status, who did not convert, who died, who were lost to follow-up or were transferred out; data for new PTB + cases and smear positive retreatment cases were recorded separately. The PTB patients in this study form around $70 \%$ of new smearpositive PTB cases and about $75 \%$ of re-smear-positive PTB treatment cases diagnosed nation-wide in the study period.

Additionally data on health facility characteristics were collected through structured interviews with the heads of the health facilities: the type (hospital/health centre), the location (urban/rural), the ownership status (private, mission, government), the highest level of health care workers in the TB department, and the date of the most recent on-the-job training for staff in the TB department. The educational levels of health care workers included university graduate (grade A0), registered nurse (grade A1), ordinary nurse (grade A2), and auxiliary nurse (grade A3). Refresher training courses on TB clinical care were considered as on-the-job-training.

Data entry was done with Epilnfo version 3.3 (CDC, Atlanta, USA) and analysis with Stata 11 (StataCorp, College Station, Texas, USA).

\section{Statistical Analysis}

The coverage of sputum microscopy for PTB + cases at the end of the 2-month intensive phase (smear completion rate) was calculated as follows. The denominator was the number of new PTB + cases initially registered minus those who had died or had been transferred out during the first two months of treatment. The numerator was the number of new PTB + cases in whom a sputum examination at $\mathrm{C} 2$ was done [10]. The SCR was calculated as the proportion of patients who had become smear-negative out of all patients of whom a 2-month sputum smear was available $[8-10,15,21,25]$. Thus, the SCR can vary between 0 and 1 . Smear completion rate and SCR were calculated similarly for retreatment cases, except that the period between start of treatment and 
expected smear examination was three rather than two months.

Because the distributions of smear completion rates and SCRs were non-normal and logit transformation could not redress this, linear regression with smear completion and SCR as outcomes was not done. Instead categorical variables were created based on cut-offs of completion rate and SCR. A completion rate of less than $90 \%$ in a health facility was regarded as a poor completion rate. An SCR of less than $75 \%$ was regarded as a low SCR [5]. Logistic regression analyses were done to assess the associations between health facility characteristics and low smear completion rate and low SCR. The natural logarithm of the total number of new TB patients in each health facility was used as a weight in the regression analyses, giving more weight to health facilities with more patients. Odds ratios were derived with 95\% confidence intervals and $\mathrm{P}$ values of $<0.05$ were considered statistically significant.

\section{Ethics}

The Rwanda National TB Control Program (PNILT) approved this study. All heads of selected health facilities agreed to participate. Informed consent of patients was not sought, as we collected and analysed anonymised aggregated routine program data.

\section{Results}

\section{New cases}

The total number of registered new PTB + cases between January and June 2006 in the 89 health facilities was 1509. Overall 1039 (69\%) of the 1509 new PTB + cases had sputum microscopy done after two months of intensive phase treatment (Table 1). As smear results were not expected from patients who had died $(n=32)$ or who had transferred out to another clinic $(n=178)$, the completion rate was $80.0 \%(1039 / 1299)$; this varied between $17.6 \%$ and $100 \%$ per health facility (median 90.6\%). Thirty-three sites had $100 \%$ coverage.

Sputum conversion was observed in $82.0 \%(852 / 1039)$ of the patients. The SCR among new PTB + cases varied per health centre between 0 and $100 \%$ with a median of 84.0\% (IQR 67.9-95.9\%). At 21 sites all examined cases had converted.

Figure 1 shows the variation of completion rate and SCR in all health facilities. Health facilities that performed well on one indicator did not necessarily perform well on the other indicator.

\section{Retreatment cases}

The total number of smear-positive retreatment patients was 206. After the three months intensive phase, a sputum smear was available for 133 out of the 176 in whom it should have been done (completion rate $75.6 \%$ ). Smear conversion was observed in 109 (82.0\%); 24 had not converted (Table 1).

\section{Health facilities}

Out of the 89 health facilities included in the study, 70 (78.7\%) were health centres and 19 (21.3\%) were hospitals. The number of newly registered PTB + cases per health facility in the six-month period varied between 0 and 71 (median 8). One site did not have any new cases and was excluded from further analyses. The number of registered retreatment cases varied between 0 and 29 (median 1). Forty-one sites did not register any retreatment cases in the study period.

Analysis of associations between health facility attributes and completion rate among new cases

Forty-three (48.9\%) health facilities had completion rates below $90 \%$. In bivariable analysis, a completion rate

Table 1 Sputum conversion rate against sputum completion rate among new TB cases for 89 health facilities

\begin{tabular}{lcc}
\hline & $\begin{array}{c}\text { New } \\
\text { patients }{ }^{\mathbf{a}}\end{array}$ & $\begin{array}{c}\text { Retreatment } \\
\text { patients }\end{array}$ \\
\hline botal number of patients & 1509 & 206 \\
No sputum examination expected after intensive phase & 210 & 30 \\
$\quad$ Died $<2$ months & $32(15.2 \%)$ & $5(16.7 \%)$ \\
Transferred out $<2$ months & $178(84.8 \%)$ & $25(83.3 \%)$ \\
Sputum examination expected after intensive phase & 1299 & 176 \\
Sputum smear done & $1039(80.0 \%)$ & $133(75.6 \%)$ \\
Sputum smear not done & $260(20.0 \%)$ & $43(24.4 \%)$ \\
Sputum smear done after intensive phase & 1039 & 133 \\
$\quad$ Sputum smear negative & $852(82.0 \%)$ & $109(82.0 \%)$ \\
Sputum smear positive & $187(18.0 \%)$ & $24(18.0 \%)$ \\
\hline
\end{tabular}

\footnotetext{
${ }^{a}$ Duration of intensive phase is 2 months.
}

${ }^{b}$ Duration of intensive phase is 3 months. 


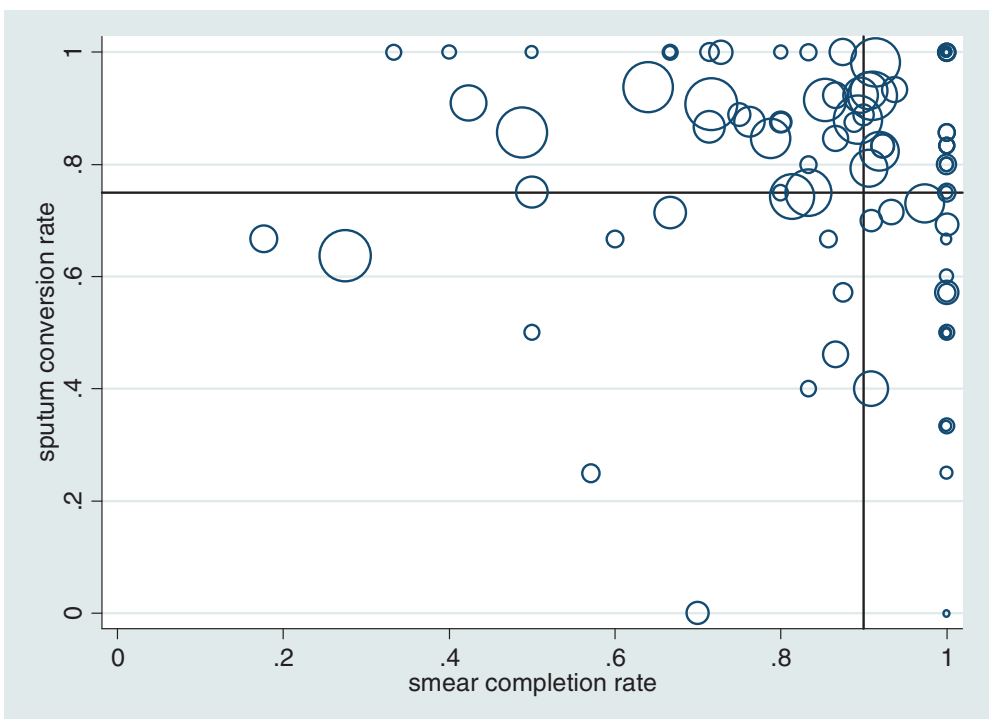

Figure 1 Sputum conversion rate against sputum completion rate among new TB cases for $\mathbf{8 9}$ health facilities. The size of the circles is proportional to the number of new TB cases diagnosed at each of the 89 health facilities in Rwanda. The horizontal line at 0.75 indicates the cut-off for an adequate sputum conversion rate. The vertical line at 0.9 indicates the cut-off for an adequate smear completion rate.

below $90 \%$ was associated with hospital (versus health centre) $(\mathrm{p}=0.025)$, urban location of facility $(\mathrm{p}=0.008)$, the province $(\mathrm{p}=0.045)$, and the number of new patients diagnosed at the centre $(\mathrm{p}=0.001)$ (Table 2$)$. In multivariable logistic regression, the only significant predictor of a low completion rate was a high number $(\geq 15)$ of new PTB cases at the health facility.

\section{Analysis of associations between health facility attributes and SCR among new cases}

Twenty-eight (31.8\%) of health facilities had smear conversion rates below $75 \%$. In bivariable analysis hospitals (versus health centres) and urban health facilities (versus rural health facilities) were less likely to have low SCR $(\mathrm{p}=0.017$ and $\mathrm{p}=0.001$, respectively; Table 3$)$. In multivariable logistic regression the only independent factor associated with a low SCR was rural location of the health facility $(\mathrm{p}=0.001)$.

\section{Discussion}

The SCR among new PTB + cases after the intensive phase of treatment was $82 \%$ among both new and retreatment cases. The WHO standard for a well functioning national TB program is a conversion rate of at least $75 \%$ among new PTB+ [5], so the Rwandan program appears to be performing well in this respect.

Comparisons of SCR between studies are challenging because different researchers have used different definitions of SCR. Some include all newly diagnosed smear-positive PTB patients in the denominator [18-20], while others only include those of whom a smear result was available after the intensive phase of treatment [7-9,15,21,25]. The first approach can yield a relatively low SCR if the program is not successful in performing smears after the intensive phase; in those cases the SCR becomes hard to interpret. Therefore we opted to calculate both a smear completion rate (reflecting the ability of the program to get sputum smears done) and the SCR (reflecting the ability of treatment to sterilise the sputum).

The SCR among new PTB patients in our study is lower than those found in studies performed in Tanzania (98.6\%) [21], China (95.0\%) [8], Cameroon (86.6\%) [9], but higher than in a refugee camp in Thailand $(75.0 \%)$ [7] and Taiwan (80.0\%) [25]. The SCR is similar to that observed in Burkina Faso (92.1\%) [10] and Uganda (76.0\%) [15].

A number of studies examined patient level factors in relation to sputum conversion. Important factors were shown to be higher pre-treatment sputum grade $[9,15-17,19,22,23]$ and extensive disease involvement on chest X-Ray $[19,20]$. Some studies found that older age $[9,16,19]$ and male sex $[10,18,21]$ were related to failure to convert. The effect of initial drug resistance on sputum conversion differed between studies [13,15,16,22,23] Co-infection with HIV has not been reported to be a significant factor for SCR or time to conversion [14-16,21,23]. So, individual patient level factors are important determinants of SCR. However in our study, we examined whether health facility factors were associated with SCR, using aggregate data. Sputum conversion was significantly associated with the location of the health facility at which patients were treated: urban sites more often had 
Table 2 Associations between poor sputum completion rates among new sputum-smear positive PTB patients and health facility characteristics at 88 health facilities, Rwanda, 2006

\begin{tabular}{|c|c|c|c|c|c|c|c|c|}
\hline & Number (\%) & $\begin{array}{l}\text { Number (\%) with } \\
\text { smear completion } \\
\text { rate }<90 \%\end{array}$ & $\begin{array}{l}\text { Bivariable } \\
\text { Odds Ratio a }\end{array}$ & $95 \% \mathrm{Cl}$ & $P^{b}$ & $\begin{array}{l}\text { Multi-variable } \\
\text { Odds Ratio }^{\text {a }}\end{array}$ & $95 \% \mathrm{Cl}$ & $P^{b}$ \\
\hline Overall & $88(100 \%)$ & $43(48.9 \%)$ & & & & & & \\
\hline Category of health facility & & & & & 0.025 & & & \\
\hline Health centre & $69(78.4 \%)$ & $30(43.5 \%)$ & 1 & & & & & \\
\hline Hospital & $19(21.6 \%)$ & $13(68.4 \%)$ & 2.0 & $1.1-3.7$ & & & & \\
\hline Location of health facility & & & & & 0.008 & & & \\
\hline Rural & $60(68.2 \%)$ & $25(41.7 \%)$ & 1 & & & & & \\
\hline Urban & $28(31.8 \%)$ & $18(64.3 \%)$ & 2.1 & $1.2-3.8$ & & & & \\
\hline Health facility status & & & & & 0.311 & & & \\
\hline Mission/private & $40(45.5 \%)$ & $19(47.5 \%)$ & 1 & & & & & \\
\hline Public & $48(54.5 \%)$ & $24(50.0 \%)$ & 1.3 & $0.8-2.3$ & & & & \\
\hline \multicolumn{9}{|l|}{$\begin{array}{l}\text { Most recent on-the-job training } \\
\text { for TB staff }\end{array}$} \\
\hline Before 2006 or none at all & $47(53.4 \%)$ & $22(46.8 \%)$ & 1 & & 0.643 & & & \\
\hline 2006 & $41(46.6 \%)$ & $21(51.2 \%)$ & 1.1 & $0.7-2.0$ & & & & \\
\hline Location of facilities (Province) & & & & & 0.045 & & & \\
\hline East & $13(14.8 \%)$ & $8(61.5 \%)$ & 1 & & & & & \\
\hline Kigali & $17(19.3 \%)$ & $11(64.7 \%)$ & 1.4 & $0.6-3.6$ & & & & \\
\hline North & $18(20.5 \%)$ & $4(22.2 \%)$ & 0.4 & $0.1-1.0$ & & & & \\
\hline West & $22(25.0 \%)$ & 11 (50.0\%) & 0.9 & $0.3-2.2$ & & & & \\
\hline South & 18 (20.5\%) & 9 (50.0\%) & 1.0 & $0.4-2.5$ & & & & \\
\hline $\begin{array}{l}\text { Number of new PTB cases in } \\
6 \text {-month period }\end{array}$ & & & & & 0.001 & & & 0.001 \\
\hline $1-5$ & 32 (36.4\%) & 9 (28.1\%) & 1 & & & 1 & & \\
\hline $6-14$ & 27 (30.7\%) & 14 (51.9\%) & 2.0 & $0.9-4.4$ & & 2.0 & $0.9-4.4$ & \\
\hline$\geq 15$ & 29 (33.0\%) & $20(69.0 \%)$ & 4.1 & $1.9-8.8$ & & 4.1 & $1.9-8.8$ & \\
\hline
\end{tabular}

a. The natural log of the number of newly registered PTB cases was used as weight in the regression analysis; ${ }^{\mathrm{b}}$ Based on the log likelihood ratio. One site did not register any new cases during the study period and was not included in the results in this table. PTB pulmonary tuberculosis; TB tuberculosis; $C I$ confidence interval; HCW health care worker.

adequate SCRs than rural sites. Underlying factors could be proximity and lower travel cost [26-28]. Lower socioeconomic status [27-29], illiteracy or insufficient knowledge about TB disease [28-30] are more frequent in rural areas. Although quality control for smear microscopy is done by the National Reference Laboratory, it is possible that the variation in SCR between centres is caused by varying quality of the reading of smears.

Failure to become smear-negative might be due to (multi-) drug-resistant TB [22]. In Rwanda a national representative study conducted in 2004-5 estimated a prevalence of $3.9 \%$ of MDR among new smear-positive PTB patients [31]. This is a relatively high prevalence, but too low to significantly impact on the SCR in Rwanda.

The completion rate of sputum smear examinations after the intensive phase of treatment was $80 \%$, which is low. Health facilities with a high number of new TB patients were more likely to have a low completion rate; this can be attributed to heavy workload in facilities that are under-staffed. District hospitals in Rwanda serve as referral facilities for all satellite diagnostic and treatment centres in their respective catchment zones. In addition to receiving many patients in routine care, these hospitals receive complicated clinical cases from health centres, including TB cases; this makes the workload heavier. Although our study did not find significant associations between level of education of health staff and completion rates, upgrading the level of education for existing staff or additional recruitments of some A1 staff at health facilities may improve smear completion rates and other standards of care. The majority of health facility staff did not get regular technical supervision or regular on-the-job training for TB management. Results 
Table 3 Associations between poor sputum smear conversion rates among new sputum-smear positive PTB patients and health facility characteristics in 88 health facilities, Rwanda, 2006

\begin{tabular}{|c|c|c|c|c|c|c|c|c|}
\hline & Number (\%) & $\begin{array}{l}\text { Number (\%) } \\
\text { with smear } \\
\text { conversion } \\
<75 \% \\
\end{array}$ & $\begin{array}{l}\text { Bivariable } \\
\text { Odds Ratio a }\end{array}$ & $95 \% \mathrm{Cl}$ & $P^{b}$ & $\begin{array}{l}\text { Multi-variable } \\
\text { Odds Ratio }^{\text {a }}\end{array}$ & $95 \% \mathrm{Cl}$ & $P^{b}$ \\
\hline Overall & $88(100 \%)$ & $28(31.8 \%)$ & & & & & & \\
\hline Category of health facility & & & & & 0.017 & & & \\
\hline Health centre & $69(78.4 \%)$ & $24(34.8 \%)$ & 1 & & & & & \\
\hline Hospital & $19(21.6 \%)$ & $4(21.1 \%)$ & 0.4 & $0.2-0.9$ & & & & \\
\hline Location of health facility & & & & & 0.001 & & & 0.001 \\
\hline Rural & $60(68.2 \%)$ & $23(38.3 \%)$ & 1 & & & 1 & & \\
\hline Urban & $28(31.8 \%)$ & $5(17.9 \%)$ & 0.3 & $0.2-0.6$ & & 0.3 & $0.2-0.6$ & \\
\hline Health facility status & & & & & 0.216 & & & \\
\hline Mission/private & $40(45.5 \%)$ & $13(32.5 \%)$ & 1 & & & & & \\
\hline Public & $48(54.5 \%)$ & $15(31.3 \%)$ & 0.7 & $0.4-1.2$ & & & & \\
\hline \multicolumn{9}{|l|}{$\begin{array}{l}\text { Most recent on-the-job } \\
\text { training for TB staff }\end{array}$} \\
\hline Before 2006 or none at all & $47(53.4 \%)$ & $15(31.9 \%)$ & 1 & & 0.235 & & & \\
\hline 2006 & $41(46.6 \%)$ & $13(31.7 \%)$ & 1.4 & $0.8-2.6$ & & & & \\
\hline Location of facilities (Province) & & & & & 0.729 & & & \\
\hline East & $13(14.8 \%)$ & $4(30.8 \%)$ & 1 & & & & & \\
\hline Kigali & $17(19.3 \%)$ & $4(23.5 \%)$ & 0.6 & $0.2-1.7$ & & & & \\
\hline North & $18(20.5 \%)$ & $8(44.4 \%)$ & 1.0 & $0.4-2.9$ & & & & \\
\hline West & $22(25.0 \%)$ & 7 (31.8\%) & 1.1 & $0.4-2.9$ & & & & \\
\hline South & 18 (20.5\%) & $5(27.8 \%)$ & 0.9 & $0.3-2.4$ & & & & \\
\hline $\begin{array}{l}\text { Number of new PTB cases in } \\
6 \text {-month period }\end{array}$ & & & & & 0.499 & & & \\
\hline $1-5$ & $32(36.4 \%)$ & $11(34.4 \%)$ & 1 & & & & & \\
\hline $6-14$ & 27 (30.7\%) & 9 (33.3\%) & 1 & $0.4-2.2$ & & & & \\
\hline$\geq 15$ & 29 (33.0\%) & $8(27.6 \%)$ & 0.7 & $0.3-1.5$ & & & & \\
\hline
\end{tabular}

The data in this table are based on new sputum smear positive patients of whom a control sputum smear was available after 2 months of intensive treatment. One site did not register any new cases during the study period and was not included in the results in this table. ${ }^{\mathrm{a}}$ The natural log of the number of newly registered PTB cases was used as weight in the regression analysis. ${ }^{b}$ Based on the log likelihood ratio. PTB pulmonary tuberculosis; TB tuberculosis; CI confidence interval; HCW health care worker.

may improve through regular on-the-job training for health care workers. Health facilities that showed a high smear completion rate did not necessarily have a high conversion rate and vice versa; very few clinics had low rates on both measures. These measures are influenced by different health care factors: the smear completion rate can be influenced by quality of the work of the clinical staff, and the conversion rate by the quality of the laboratory work.

This study has some limitations. The data were collected in 2006 and possibly smear completion rate has improved since then. The sample size of this study was limited, although this was a representative sample of all health facilities diagnosing and treating $\mathrm{TB}$, the power to detect significant associations was limited. Error in completing records may not be uncommon in routine clinics and may have led to bias in data collection. Individual patient factors like age, sex, smear grade and adherence are -or may be- related to smear conversion; this study focused on health sector factors, and thus omitted knowingly important factors from the analysis.

\section{Conclusions}

In Rwanda the coverage of smear sputum examination after the end of the intensive phase of TB treatment is too low and efforts are needed to increase this. The SCR in Rwanda at $82 \%$ is adequate. A high number of new TB patients at a health facility was a significant predictor of a low completion rate. The national TB control program should design strategies to improve completion rates, with a focus on the busiest clinics. 


\section{Competing interests}

The authors declare that they have no competing interests.

\section{Acknowledgements}

Initial data collection was funded by the Rwanda National TB Control Program (PNILT), Ministry of Health, Kigali, Rwanda. We thank Prof. Alexander Lyambabaje for statistical advice.

We thank Dr F. Cobelens, Dr L. de Naeyer, Prof. P. Klatser and Dr M. Toussaint for comments on an earlier draft of the manuscript.

Technical support was provided by the Infectious Disease Network for Treatment and Research in Africa (INTERACT), funded by the Netherlands Organization for Scientific Research/Netherlands Foundation for the Advancement of Tropical Research (NWO/WOTRO) and the European Union (SANTE/2006/105-316).

\section{Author details}

${ }^{1}$ INTERACT, CPCD, PO Box 2181, Kigali, Rwanda. ${ }^{2}$ Royal Tropical Institute, KIT Biomedical Research, Mauritskade 63, 1092 AD, Amsterdam, Netherlands. ${ }^{3}$ ICAP, Mailman School of Public Health, Columbia University, P.O. Box 3989 Kigali, Rwanda. ${ }^{4}$ Rwanda National TB Control Program (PNILT), Ministry of Health, P.O. Box 84, Kigali, Rwanda. ${ }^{5}$ Center for poverty-related communicable diseases (CPCD) and Center for Infection and Immunity Amsterdam (CINIMA), AMC, P.O. Box 22700, 1100 DE, Amsterdam, Netherlands. ${ }^{6}$ Public Health Service of Amsterdam (GGD), P.O. Box 2200, 1000 CE, Amsterdam, Netherlands.

\section{Authors' contributions}

VM, FK and MG designed the study. FK carried out data collection. FK, MB and MSvdL conducted the statistical analysis. FK wrote the first draft of the paper. All authors contributed to revisions and saw and approved the final version of the manuscript.

\section{Received: 25 November 2011 Accepted: 4 July 2012}

Published: 16 July 2012

\section{References}

1. World Health Organization: Global Tuberculosis Control 2011. WHO report 2011. Geneva: WHO; 2011. WHO/HTM/TB/2011.16.

2. World Health Organization: TB/HIV Clinical manual. Geneva: WHO; 2004 WHO/HTM/TB/2004.329.

3. Rwanda Biomedical Center (RBC). Institute of HIV/AIDS, Disease Prevention \& Control (IHDPC) TB \& Other Respiratory Communicable Diseases Division. Rwanda ministry of Health: Annual report 2010. Kigali: RBC/RMOH; 2010.

4. World Health Organization: Treatment of tuberculosis guidelines. Geneva: WHO; 2010. WHO/HTM/TB/2009.420.

5. World Health Organization: Compendium of indicators for Monitoring and Evaluating National Tuberculosis programs. Geneva: WHO; 2004. WHO/HTM/ TB/2004.344

6. American Thoracic Society/Centers of Disease Control and Prevention/ Infectious Diseases Society of America: Controlling tuberculosis in the United States. Am J Respir Crit Care Med 2005, 172:1169-1227.

7. Rieder HL: Sputum smear conversion during directly observed treatment for tuberculosis. Int J Tuberc Lung Dis 1996, 77:124-129.

8. Feng-Zeng Z, Levy MH, Wen S: Sputum microscopy results at two and three months predict outcome of tuberculosis treatment. Int J Tuberc Lung Dis 1997, 1:570-572.

9. Kuaban C, Bame R, Mouangue L, Djella S, Yomgni C: Non conversion of sputum smears in new smear positive pulmonary tuberculosis patients in Yaoundé, Cameroon. East African Medical Journal 2009, 86:219-225.

10. Dembele SM, Ouedraogo HZ, Combary A, Saleri N, Macq J, Dujardin B: Conversion rate at two-month follow-up of smear-positive tuberculosis patients in Burkina Faso. Int J Tuberc Lung Dis 2007, 11:1339-1344.

11. TRAC Plus, TB Unit, Rwanda Ministry of Health: Hand book of Tuberculosis and TB-HIV 2009. Kigali: TRAC Plus/RMOH; 2009.

12. Chaulk CP, Moore-Rice K, Rizzo R, Chaisson RE: Eleven years of communitybased directly observed therapy for tuberculosis. JAMA 1995, 274:945-951.

13. Telzak EE, Barkat AF, Pollard CL, Turett GS, Justaman JE, Blum S: Factors influencing time to sputum conversion among patients with smearpositive pulmonary tuberculosis. Clin Infect Dis 1997, 25:666-670.

14. Fortún J, Martin-Davilla P, Molina A, Navas E, Hermida JM, Cobo J, Gomez-Mampaso E, Moreno S: Sputum conversion among patients with pulmonary tuberculosis: are there implications for removal of respiratory isolation? J Antimicrob Chemother 2007, 59:794-798.

15. Bwire R, Borgdorff MW, Stitch-Groh V, Rieder HL, Kawuma HJ, Bretzel G, Rüsch-Gerdes S: Tuberculosis chemotherapy and sputum conversion among HIV-seropositive and HIV-seronegative patients in south-eastern Uganda. East Afr Med J 1999, 76:307-313.

16. Glynn JR, Warndorff DK, Fine PEM, Munthali MM, Sichone W, Ponnighaus JM: Measurement and determinants of tuberculosis outcome in Karonga District, Malawi. Bull World Health Organ 1998, 76:295-305.

17. Singla R, Osman MM, Khan N, Al-Sharif N, Al-Sayegh MO, Shaik MA: Factors predicting persistent sputum smear positivity among pulmonary tuberculosis patients 2 months after treatment. Int J Tuberc Lung Dis 2003, 7:58-64.

18. Pungrassami $P$, Johnsen SP, Chongsuvivatwong V, Olsen J: Has directly observed treatment improved outcomes for patients with tuberculosis in southern Thailand? Tropical Medicine and International Health 2002, 7:271-279.

19. Banu Rekha W, Balasubramanian R, Swaminathan S, Ramachandran R, Rahman F, Sundaram V, Thyagarajan K, Selvakumar N, Adhilakshmi AR, lliayas S, Narayanan PR: Sputum conversion at the end of intensive phase of category-1 regimen in the treatment of pulmonary tuberculosis patients with diabetes mellitus or HIV infection: An analysis of risk factors. Indian J Med Res 2007, 126:452-458.

20. Hesseling AC, Walzl G, Enarson DA, Carroll NM, Duncan K, Lukey PT, Lombard C, Donald PR, Lawrence KA, Gie RP, van Helden PD, Beyers N: Baseline sputum time to detection predicts month two culture conversion and relapse in non-HIV-infected patients. Int J Tuberc Lung Dis 2010, 14:560-570.

21. Senkoro M, Mfinanga SG, Mørkve O: Smear microscopy and culture conversion rates among smear positive pulmonary tuberculosis patients by HIV status in Dar es Salaam. Tanzania. BMC Infect Dis 2010, 10:210.

22. Horne DJ, Johnson CO, Oren E, Spitters C, Narita M: How soon should patients with smear-positive tuberculosis be released from inpatient isolation? Infect Control Hosp Epidemiol 2010, 31:78-84

23. Noeske J, Nguenko PN: Impact of resistance to anti-tuberculosis drugs on treatment outcome using World Health Organization standard regimens. Trans R Soc Trop Med Hyg 2002, 96:429-433.

24. TRAC Plus, TB Unit, Rwanda Ministry of Health: Hand book of tuberculosis and TB-HIV 2007. Kigali: TRAC Plus/RMOH; 2007.

25. Su WJ, Feng JY, Chiu YC, Huang SF, Lee YC: Role of 2-month sputum smears in predicting culture conversion in pulmonary tuberculosis. Eur Respir J 2011, 37:376-383.

26. Jaiswal A, Singh V, Ogden JA, Porter JDH, Sharma PP, Sarin R, Arora VK, Jain RC: Adherence to tuberculosis treatment: lessons from the urban setting of Delhi, India. Tropical Medicine and International Health 2003, 8:625-633.

27. Mishra P, Hansen EH, Sabroe S, Kafle KK: Socio-economic status and adherence to tuberculosis treatment: a case-control study in a district of Nepal. Int J Tuberc Lung Dis 2005, 9:1134-1139.

28. Gopi PG, Vasantha M, Muniyandi M, Chandrasekaran V, Balasubramanian R, Narayanan PR: Risk factors for non-adherence to directly observed treatment (DOT) in a rural tuberculosis unit, South India. Indian J Tuberc 2007, 54:66-70.

29. Singh V, Jaiswal A, Porter JD, Ogden JA, Sarin R, Sharma PP, Arora VK, Jain RC: TB control, poverty, and vulnerability in Delhi, India. Tropical Medicine and International Health 2002, 7:693-700.

30. Vijay S, Kumar P, Chauhan LS, Vollepore BH, Kizhakkethil UP, Rao SG: Risk factors associated with default among new smear positive TB patients treated Under DOTS in India. PLOS One 2010, 5:e10043.

31. Umubyeyi AN, Vandebriel G, Gasana M, Basinga P, Zawadi JP, Gatabazi J, Pauwels P, Nzabintwali F, Nyiramasarabwe L, Fissette K, Rigouts L, Struelens MJ, Portaels F: Results of a national survey on drug resistance among pulmonary tuberculosis patients in Rwanda. Int J Tuberc Lung Dis 2007, 11:189-194.

doi:10.1186/1756-0500-5-357

Cite this article as: Kayigamba et al:: Sputum completion and conversion rates after intensive phase of tuberculosis treatment: an assessment of the Rwandan control program. BMC Research Notes 2012 5:357. 